\title{
Effect of transition metal ions on luminescence of MOFs
}

\author{
Bo Ruan ${ }^{1,+}$, Huan-Li Liư ${ }^{1,+}$, Xue-Qing Zhan ${ }^{1}$, Hui Ding ${ }^{1}$, Lei Xie ${ }^{1}$, Fang-Chang Tsai1,* \\ School of Materials Science and Engineering, Hubei University, Wuhan 430062, China \\ + Both authors contributed equally
}

\begin{abstract}
Three kinds of metal-organic framework materials (MOF-5, MLI-101(Cr), UiO-66) are synthesized, which are based on transition metal $(\mathrm{Zn}, \mathrm{Cr}, \mathrm{Zr})$ as the center ions, and terephthalic acid $\left(\mathrm{H}_{2} \mathrm{BDC}\right)$ as organic ligand. At the same time, three kinds of MOFs were characterized and analyzed with X-ray diffraction (XRD), scanning electron microscopy (SEM), UV-visible absorption spectrometer (UV-Vis), and fluorescence spectrometer (PL). This article analyzes the fluorescence effects of these three MOFs and investigates the role of different transition metal ions in MOF luminescence. The results show that the ionic radii of $\mathrm{Zn}^{2+}$ and $\mathrm{Zr}^{4+}$ are similar, which are $0.074 \mathrm{~nm}$ and 0.072 $\mathrm{nm}$, respectively. MOF-5 and UiO-66 have similar fluorescence effects, both of which emit blue-violet light. However, MIL-101 (Cr) fluorescence centers on $\mathrm{Cr}^{3+}$ with an ionic radius of $0.0651 \mathrm{~nm}$ emits yellow-green light, which is different from the foregoing MOFs. Therefore, the ionic radius of the transition metal has an important effect on the fluorescence effect of the MOF. For larger ionic radii, the fluorescence of MOF is generally in the blue-violet square region.
\end{abstract}

\section{Introduction}

Luminescence is a very rare phenomenon, which generally refers to the luminescence ability of the molecule when the ground state electrons in the molecule absorb energy to transition to the excited state and then return to the ground state by means of radiative transition $^{[1,2]}$. Therefore, various luminescent substances have received extensive attention. As people's demand for luminescent materials continues to increase, many new functional materials have been explored and discovered. Luminescent metal-organic framework materials, due to their design of the main structure of the fusion of the two major properties of porosity and light, can show a relatively rich host-guest interaction, and thus get a wide range of attention ${ }^{[3-6]}$. The metal-organic framework material is a porous material composed of a metal ion or a metal oxygen cluster as a central node and an organic chain-like molecule ligand, which simultaneously possesses an inorganic block and an organic block, which may have luminescent properties, and the combination of inorganic blocks and organic blocks can also form unique metal-ligand intramolecular charge transfer to induce new luminescence properties ${ }^{[7-10]}$. Therefore, the metal organic framework material is a very good luminescent material.

Most of the metal elements in the periodic table of elements have been used to build MOFs, of which transition metals are very common. Therefore, it is very meaningful to study the effect of transition metals on the luminescence properties of MOFs. Here we select transition metal ions of different valences as the central ions. Zinc ions are the most common among the divalent ions which have suitable hardness and have good binding with common donor atoms such as oxygen and nitrogen ${ }^{[1-13]}$. Trivalent transition metal ions such as $\mathrm{Cr}^{3+}$ have a smaller radius and a higher charge, which can form strong coordination bonds with oxygen-containing ligands, and for tetravalent transition metal ions such as $\mathrm{Zr}^{4+}$, the coordination bond is more strong ${ }^{[14]}$. Metalorganic framework materials generally use $\pi$-conjugated organic molecules as organic ligands, which have very good rigidity, and most of them are based on functionalization with polycarboxylate groups or heterocyclic groups ${ }^{[12,13]}$. One of the most common and simplest is terephthalic acid $\left(\mathrm{H}_{2} \mathrm{BDC}\right)^{[15-17]}$. Therefore terephthalic acid is often used as an organic ligand for MOFs. Therefore, this article selects terephthalic acid as the organic ligand and uses $\mathrm{Zn}^{2+}, \mathrm{Cr}^{3+}$, and $\mathrm{Zr}^{4+}$ as the central ions to synthesize MOF-5, MIL-101(Cr) and UiO66 and study the luminescence effects of these MOFs.

\section{Experimental}

\subsection{Materials and synthesis}

All reagents were purchased commercially and used without further purification.

The synthesis of MOFs are shown in Figure 1. 
Taking MIL-101(Cr) as an example, $\mathrm{Cr}\left(\mathrm{NO}_{3}\right)_{3} \cdot 9 \mathrm{H}_{2} \mathrm{O}$ (2 mmol, $0.8054 \mathrm{~g}), \mathrm{H}_{2} \mathrm{BDC}$ (2 mmol, $0.3326 \mathrm{~g}$ ), $0.1 \mathrm{~mL}$ hydrofluoric acid (wt.\%, 35\%) and $9.6 \mathrm{~mL}$ deionized water were placed in a $50 \mathrm{ml}$ Teflon lining. Then, the inner liner was placed in a stainless steel reactor, and the lid of the reactor was placed in an oven. The temperature of the oven was increased from room temperature to $200^{\circ} \mathrm{C}$ within 3 hours and maintained at $200^{\circ} \mathrm{C}$ for 8 hours. After that, it was cooled to room temperature then filtered through a fritted funnel to give a green precipitate. The product was washed with DMF and EtOH, respectively. Then it was centrifuged for 5 minutes to obtain precipitate (revolution speed $8000 \mathrm{rpm}$ ) and repeatedly washed twice more. Finally, the suction filtration was used. The solid obtained by suction filtration was dried in a vacuum oven at $150^{\circ} \mathrm{C}$ for $12 \mathrm{~h}$. In this way, MIL-101 (Cr) used in the following experiment was prepared.

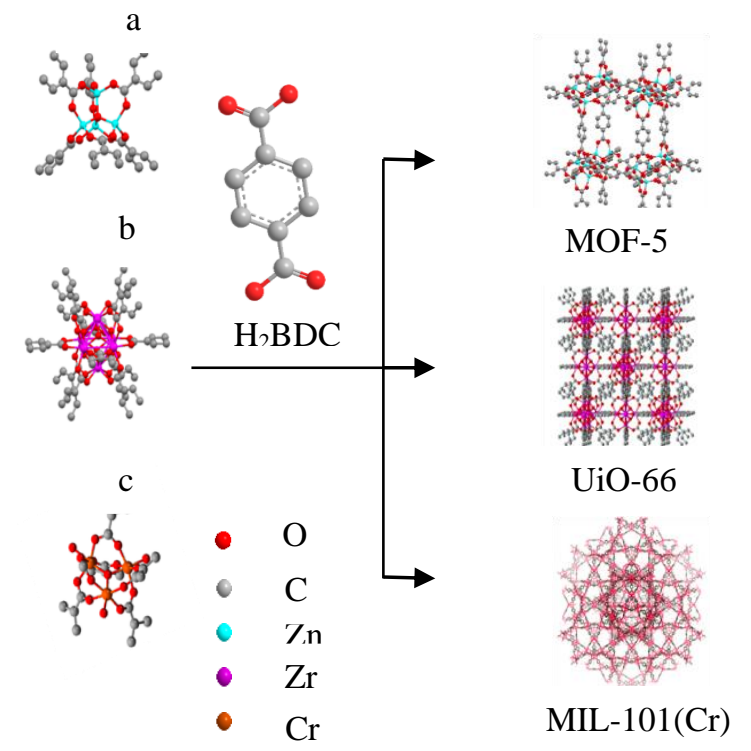

Figure 1. The concept of syntheses of MOF-5, MIL$101(\mathrm{Cr})$ and UiO-66; $\mathrm{a}, \mathrm{b}$ and $\mathrm{c}$ are SBU of $\mathrm{Zn}^{2+}, \mathrm{Zr}^{4+}$ and $\mathrm{Cr}^{3+}$, respectively.

\subsection{Characterization}

The sample powder was ground uniformly and then compacted in a mold and placed on a Bruker D8A25 Xray powder diffraction (PXRD). With a $\mathrm{Cu}$ target, $\lambda \mathrm{K} \alpha=$ $0.154 \mathrm{~nm}, \mathrm{I}=40 \mathrm{~mA}$, and $\mathrm{U}=4 \mathrm{kV}$, the test was performed in a continuous scanning mode with a scanning range of $2-40^{\circ}$, a scanning speed of $4 \mathrm{deg} / \mathrm{min}$, and a step length of 0.02 step size. The crystal morphology of MOFs was observed on a scanning electron microscope (SEM) (JSM7100F).

UV-visible absorption spectra of the prepared metalorganic framework materials were obtained with integrating sphere test mode of UV3600. And the wavelength coverage is $200 \mathrm{~nm}$ to $700 \mathrm{~nm}$. The digital photo was taken mainly under white light and $365 \mathrm{~nm} \mathrm{UV}$ light. The fluorescence emission spectra (PL) of the prepared materials were obtained using an LS-55 fluorescence spectrophotometer from Perkin Elmer, USA. The entire test was performed at room temperature with an applied voltage of $650 \mathrm{~V}$ and a test step of $0.5 \mathrm{~nm}$.
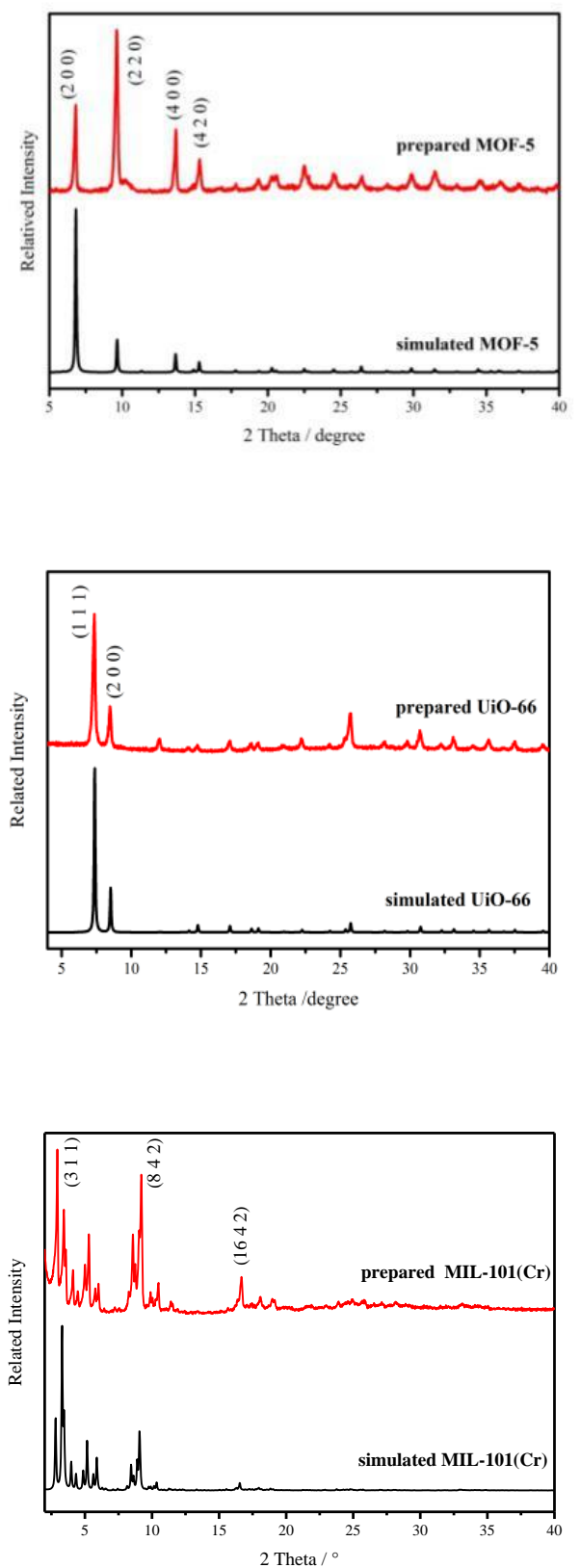

Figure 2. PXRD of MOF-5, UiO-66, and MIL-101(Cr)

\section{Results and discussion}

\subsection{Powder X-ray diffraction (PXRD)}

MOFs have the characteristics of both inorganic and organic materials. The specific crystal structures of the MOFs are generally determined by powder X-ray diffraction analyses. Decrystallization of the crystal CIF 
file can then lead to an X-ray diffraction pattern that can be used to evaluate whether or not the same structural MOFs were prepared. The polycrystalline powder X-ray diffraction of MOFs herein are shown in Figure 2. Through the analyses of the maps, we can find that the location and relative intensity of as-synthesized MOFs diffraction peaks are consistent with those of the simulation. These show that MOFs were successfully prepared.
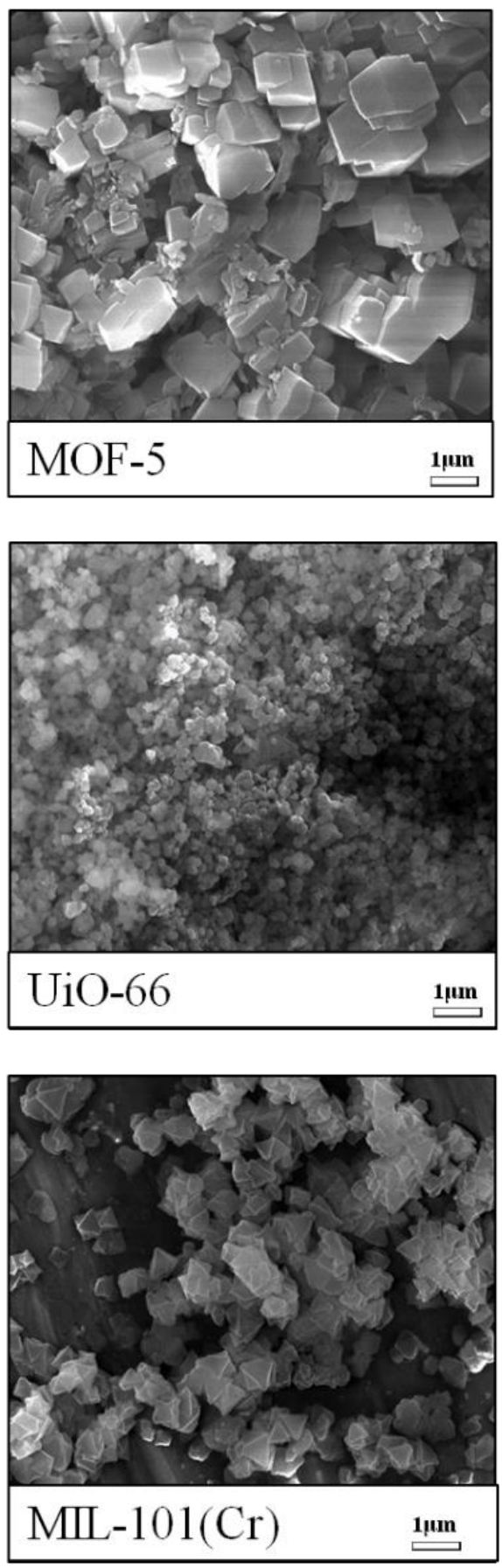

Figure 3. SEM images of MOF-5, UiO-66 and MIL$101(\mathrm{Cr})$

\subsection{Scanning electron microscope (SEM)}

Scanning electron microscope images of metal-organic frameworks are shown in Figure 3. MIL-101 (Cr) has a cage topology with MTN molecular sieves with a regular octahedral crystal morphology. The crystal morphology of UiO-66 is a particularly small granular morphology. The morphology of MOF-5 prepared by the mechanical method is a stack structure of various block structures Although the MOFs have the same ligand, the crystal morphology of the MOFs is different due to the different transition metal ions. Therefore, we can speculate that the central ion plays an important role in the formation of crystals in MOFs, which further affects the luminescence properties of MOFs.

\subsection{Luminescence properties}

The UV absorption and fluorescence emission spectra of the MOFs are shown in Figure 4. The UV absorption peaks and fluorescence emission peaks of the MOFs are shown in Table 1. UiO-66 was excited by light with the excitation wavelength of $330 \mathrm{~nm}$. The complex produces a single-peak emission of 350-500 nm. The main emission band was at $398.5 \mathrm{~nm}$, which was attributed to the internal $\pi-\pi^{*}$ charge transition (ILCT) of the ligand. Also UV absorption peak of UiO-66 is a broad singlet of 200-350 nm. With it excited at an excitation wavelength of $338 \mathrm{~nm}$, MOF-5 produces a single emission of 400-500 $\mathrm{nm}$. The most important emission band is at $440 \mathrm{~nm}$, which is attributed to the $\pi-\pi^{*}$ charge transition (ILCT) inside the ligand. UV absorption peak of MOF-5 is a broad singlet of $200-400 \mathrm{~nm}$. The MIL-101 (Cr) was excited by light at a wavelength of $395 \mathrm{~nm}$. The complex produces three emission peaks at $485 \mathrm{~nm}, 525 \mathrm{~nm}$, and $580 \mathrm{~nm}$. The first peak is attributed to the ligand's internal $\pi-\pi^{*}$ charge transition (ILCT), and the latter two peaks are attributed to electronic transitions between the internal energy levels of the metal ion. UV absorption of MIL$101(\mathrm{Cr})$ is $200-700 \mathrm{~nm}$ full-spectrum absorption. In Table 2 , we can see that the original white color of MOF-5 is changed to blue-violet under the irradiation of ultraviolet light. This means that MOF-5 emits blue-violet light. At the same time, under the irradiation of ultraviolet light, white color of UiO-66 becomes blue-purple, which shows that UiO-66 emits blue-violet light. MIL-101 (Cr) changes from blue-green to yellow-green under irradiation of ultraviolet light, which indicates that it emits yellow-green light.

For MOF-5, it has been studied that the luminescence of MOF-5 is derived from carboxylic acid to zinc ion charge transfer (LMCT), but theoretical calculations and spectroscopic studies have shown that MOF-5 luminescence is more likely to be based on internal ligand transitions $^{[18,19]}$. In Figure 4 we can see that terephthalic acid itself is not fluorescent. And why is the emission of MOF-5 caused by terephthalic acid when terephthalic acid is combined with zinc ions to form MOF-5? This may be because the combination of terephthalic acid and metal ions to form MOF enhances the intermolecular force, which makes the excited state molecules more susceptible to optical radiation and thus enhances fluorescence. At the same time, the outermost electron of 
zinc ion is the structure of $\mathrm{d}^{10}$, which is in a state of full filling. This means that the outer electrons of the zinc ion are very stable and do not easily undergo electron transitions. As a result, the terephthalic acid ligand can emit light.

Table 1. UV absorption peak and fluorescence emission peak of $\mathrm{H}_{3} \mathrm{BDC}$, MOF-5,UiO-66 and MIL-101(Cr)

\begin{tabular}{ccc}
\hline & \multicolumn{1}{c}{$\begin{array}{c}\text { Ultraviolet } \\
\text { absorption peak }\end{array}$} & $\begin{array}{c}\text { Flurescent } \\
\text { emission peak }\end{array}$ \\
\hline $\begin{array}{c}\text { Terephthalic } \\
\text { acid }\end{array}$ & $200-350 \mathrm{~nm}$ & $※$ \\
MOF-5 & $200-400 \mathrm{~nm}$ & $440 \mathrm{~nm}$ \\
UiO-66 & $200-400 \mathrm{~nm}$ & $398 \mathrm{~nm}$ \\
MIL-101(Cr) & $200-700 \mathrm{~nm}$ & $\begin{array}{c}485 \mathrm{~nm}, 525 \mathrm{~nm}, \\
580 \mathrm{~nm}\end{array}$ \\
& & \\
\hline
\end{tabular}

$※$ means no fluorescence

Table 2. Photograph of three kinds of MOF under white light and ultraviolet light

\begin{tabular}{lll}
\hline & $\begin{array}{l}\text { Nature } \\
\text { light }\end{array}$ & $\begin{array}{l}\text { Ultraviolet } \\
\text { light/365nm }\end{array}$ \\
\hline MOF-5 & & \\
UiO-66 & & \\
MIL-101(Cr) & & \\
\hline
\end{tabular}

Similarly, we can find that the outermost electron of zirconium ion is the structure of $\mathrm{d} 0$, and it is in the empty state. This means that the outer electrons of the zirconium ion are also very stable and electron transitions are unlikely to occur. UiO-66, like MOF-5, has an organic linker ligand that is also terephthalic acid. Therefore, we can speculate that UiO-66, like MOF-5, emits light because it is based on the internal $\pi-\pi^{*}$ electron transition of the organic ligand. Meanwhile, we can find that UiO66 and MOF-5 have similar UV absorption and fluorescence emission in Figure 4. This further shows that UiO-66 emits light based on the $\pi-\pi^{*}$ charge transition inside the ligand. For MIL-101(Cr), although the organic ligand of MIL-101(Cr) is also terephthalic acid, its luminescence mechanism is different from that of MOF-5. Since the outermost electron of the chromium ion is the
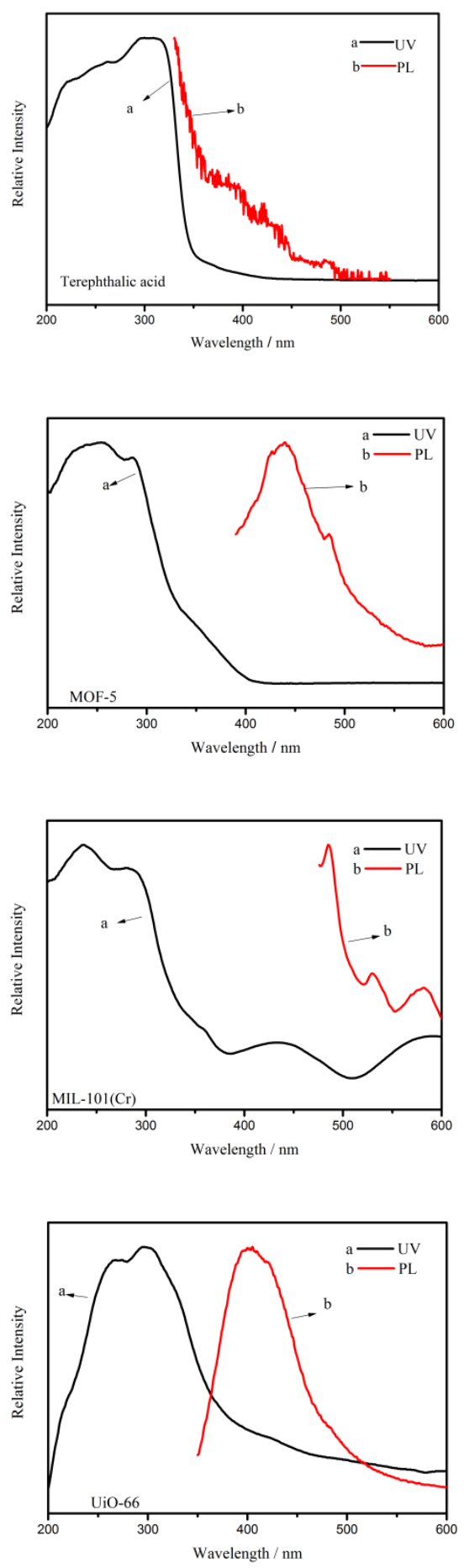

Figure 4. UV absorption and fluorescence emission spectra of terephthalic acid $\left(\mathrm{H}_{2} \mathrm{BDC}\right), \mathrm{MOF}-5$, UiO-66 and MIL-101(Cr)

structure of $\mathrm{d}^{3}$, this means that the outer electrons of the chromium ion are very active and are prone to electronic transitions. So we can speculate that chromium ions play an important role in the luminescence of MOL-101(Cr). 
From Figure 5, we can see that the ground state of $\mathrm{Cr}^{3+}$ is ${ }^{4} \mathrm{~F}$. In the octahedral symmetric crystal field, ${ }^{4} \mathrm{~F}$ splits into ${ }^{4} \mathrm{~A}_{2}$ (ground state), ${ }^{4} \mathrm{~T}_{2}$ and ${ }^{4} \mathrm{~T}_{1}$; Excited ${ }^{4} \mathrm{P}$ splits into ${ }^{4} \mathrm{~T}_{1} .{ }^{2} \mathrm{G}$ splits into ${ }^{2} \mathrm{E},{ }^{2} \mathrm{~T}_{1}$ and ${ }^{2} \mathrm{~T}_{2} .{ }^{2} \mathrm{~F}$ splits into ${ }^{2} \mathrm{~A}_{2}$. In Figure 4 we can see that the ultraviolet absorption spectrum of MIL-101(Cr) is composed of four main absorption peaks. According to the spin selection rule of the optical transition $\triangle S=0$, transitions from the ground state ${ }^{4} \mathrm{~A}_{2}$ to ${ }^{4} \mathrm{~T}_{2},{ }^{4} \mathrm{~T}_{1}\left({ }^{4} \mathrm{~F}\right)$ and ${ }^{4} \mathrm{~T}_{1}\left({ }^{4} \mathrm{P}\right)$ are allowed. The absorption peak at $273 \mathrm{~nm}$ comes from the absorption of terephthalic acid. The absorption peaks at $237 \mathrm{~nm}, 431$ $\mathrm{nm}$, and $588 \mathrm{~nm}$ are all generated by $\mathrm{Cr}^{3+}$, which are respectively caused by the energy level transitions of ${ }^{4} \mathrm{~A}_{2-}$ ${ }^{4} \mathrm{~T}_{1}\left({ }^{4} \mathrm{P}\right)$ and ${ }^{4} \mathrm{~A}_{2}{ }^{4} \mathrm{~T}_{1}\left({ }^{4} \mathrm{~F}\right)$ and ${ }^{4} \mathrm{~A}_{2-}{ }^{4} \mathrm{~T}_{2}$ in $\mathrm{Cr}^{3+}$. In Figure 4 we can see that there is a large overlap between the UV absorption spectrum of MIL-101(Cr) and the UV absorption spectrum of terephthalic acid, which provides plenty of possibilities that energy is transferred from terephthalic acid to chromium ions. In Figure 4 we can see that the fluorescence emission spectrum of MIL$101(\mathrm{Cr})$ consists of three emission peaks. We can speculate that the emission peak at $485 \mathrm{~nm}$ is caused by the charge transition of terephthalic acid to $\mathrm{Cr}^{3+}$. The emission peak at $525 \mathrm{~nm}$ is generated by the ${ }^{2} \mathrm{E}-{ }^{4} \mathrm{~A}_{2}$ transition of $\mathrm{Cr}^{3+}$. The emission peak at $580 \mathrm{~nm}$ is generated by the ${ }^{4} \mathrm{~T}_{2-}{ }^{4} \mathrm{~A}_{2}$ transition of $\mathrm{Cr}^{3+}$.

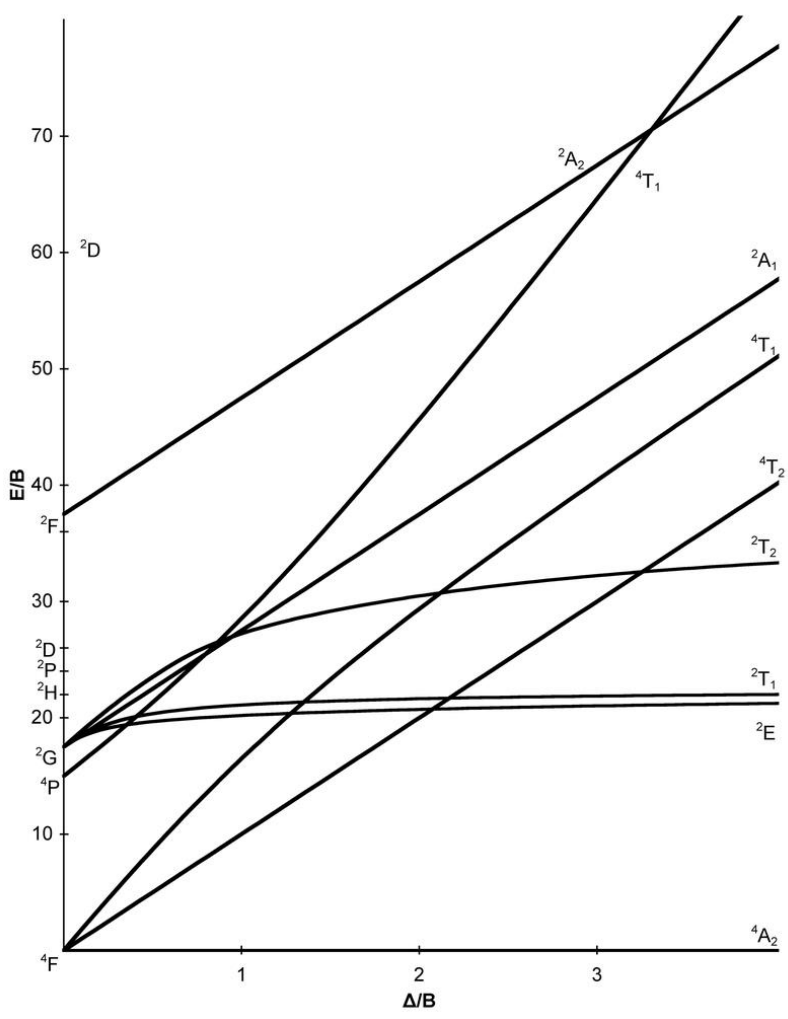

Figure 5. Crystal field splitting Tanabe-Sugano diagram of transition metal $\mathrm{Cr}^{3+}$. ${ }^{[20]}$

Although the organic ligands are terephthalic acid, the three MOFs have different fluorescence effects due to their different transition metal ions. After consulting the literature, the radius of zirconium ions is $0.072 \mathrm{~nm}$, the radius of chromium ions is $0.0615 \mathrm{~nm}$, and the radius of zinc ions is $0.074 \mathrm{~nm}$. We can find that the radius of zirconium ion is similar to the radius of zinc ion. At the same time, it can be seen from Figure 6 that the fluorescence emitted by MOF-5 and UiO-66 is mainly

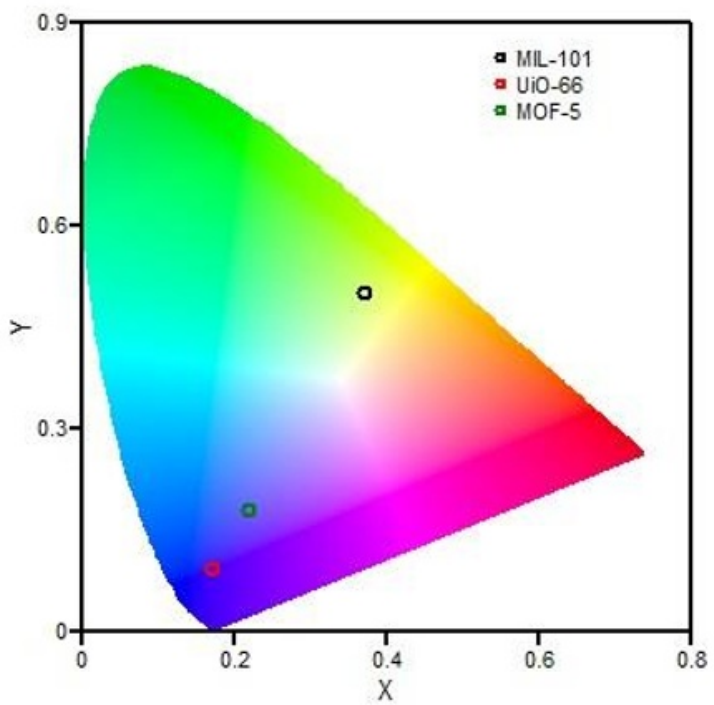

Figure 6. Three kinds of MOF chromaticity diagram

distributed in the blue-violet region of the chromaticity diagram, while the fluorescence emitted by MIL-101 (Cr) is mainly distributed in the yellow-green region of the chromaticity diagram, which is consistent with the previous analysis. We can find that MOF-5 with similar ionic radius has a similar fluorescence effect as UiO-66, which is different from the fluorescence effect of MIL$101(\mathrm{Cr})$ with a smaller ion radius. Similarly, Wang and colleagues found that the observation of LMCT or linker luminescence depends on the metal ion radius in transition metal-containing $\mathrm{MOFs}^{[21]}$. Therefore, it is speculated that when the organic ligands are the same, the radius of the transition metal ions plays an important role in the luminescence of the MOF.

\section{Conclusions}

MOF-5, UiO-66, and MIL-101 (Cr) have been successfully synthesized. All three MOFs have a strong fluorescence effect, in which the fluorescence emission spectra of both MOF-5 and UIO-66 are relatively broad and emit blue-violet light, while the fluorescence emission spectrum of MIL-101 (Cr) is a multimodal fluorescence emission with multiple excited states. In addition, MOF-5 and UiO-66 with the similar transition metal ion radii have similar fluorescence effects, while the fluorescence of MIL-101(Cr) with smaller transition metal ion radius is very different from the former two MOFs. Therefore, the luminescence of the MOF is closely related to the transition metal ion radius in the MOF. 


\section{Acknowledgement}

This study has been supported by the National High Technology Research and Development Program (863 program) of China No. 2012AA06A111; Hubei Provincial Natural Science Foundation of China No. 2014CFB552; Hubei Collaborative Innovation Center for Advanced Organic Chemical Materials of China No. 00001647909 .

\section{References}

1. M. D. Allendorf, C. A. Bauer, R. K. Bhakta and R. J. Houk, Chemical Society reviews, 38, 1330-1352 (2009).

2. V. Amendola, L. Fabbrizzi, F. Foti, M. Licchelli, C. Mangano, P. Pallavicini, A. Poggi, D. Sacchi and A. Taglietti, Coordination Chemistry Reviews, 250, 273-299 (2006).

3. R. H. Kyriakos C. Stylianou, Samantha Y. Chong, John Bacsa,, Y. Z. K. James T. A. Jones, Darren Bradshaw, * and and M. J. Rosseinsky, J. AM. CHEM. SOC., 132, 4119-4130 (2010).

4. Banglin Chen, $\dagger$ Liangbo Wang, $\uparrow$ Fatima Zapata, $\uparrow$ Guodong Qian,*, ${ }^{*}$ and Emil B. Lobkovsky§, J. AM. CHEM. SOC, 130, 6718-6719 (2008).

5. Z. Hu, B. J. Deibert and J. Li, Chemical Society reviews, 43, 5815-5840 (2014).

6. L. E. Kreno, K. Leong, O. K. Farha, M. Allendorf, R. P. Van Duyne and J. T. Hupp, Chemical reviews, 112, 1105-1125 (2012).

7. Y. Cui, B. Li, H. He, W. Zhou, B. Chen and G. Qian, Accounts of chemical research, 49, 483-493 (2016).

8. R. J. Kuppler, D. J. Timmons, Q.-R. Fang, J.-R. Li, T. A. Makal, M. D. Young, D. Yuan, D. Zhao, W. Zhuang and H.-C. Zhou, Coordination Chemistry Reviews, 253, 3042-3066 (2009).

9. N. Stock and S. Biswas, Chemical reviews, 112, 933969 (2012).

10. H. C. Zhou, J. R. Long and O. M. Yaghi, Chemical reviews, 112, 673-674 (2012).

11. P. Silva, S. M. Vilela, J. P. Tome and F. A. Almeida Paz, Chemical Society reviews, 44, 6774-6803 (2015).

12. M. Yu, M. Hu and Z. Wu, RSC Advances, 3, 25175 (2013).
13. Y. Cui, Y. Yue, G. Qian and B. Chen, Chemical reviews, 112, 1126-1162 (2012).

14. T. Devic and C. Serre, Chemical Society reviews, 43, 6097-6115 (2014).

15. D. Jiang, A. D. Burrows and K. J. Edler, CrystEngComm, 13, 6916 (2011).

16. M. Kandiah, M. H. Nilsen, S. Usseglio, S. Jakobsen, U. Olsbye, M. Tilset, C. Larabi, E. A. Quadrelli, F. Bonino and K. P. Lillerud, Chemistry of Materials, 22, 6632-6640 (2010).

17. W. J. Son, J. Kim, J. Kim and W. S. Ahn, Chemical communications, 6336-6338 (2008).

18. M. Ji, X. Lan, Z. Han, C. Hao and J. Qiu, Inorganic chemistry, 51, 12389-12394 (2012).

19. P. Feng, J. Perry Iv, S. Nikodemski, B. Jacobs, S. Meek and M. Allendorf, Journal of the American Chemical Society, $\mathbf{4 4 , 2 0 1 0 . ~}$

20. T. U. o. C. Davis, University of California Davis, 2010 .

21. Y.-T. Wang, G.-M. Tang and Z.-W. Qiang, Polyhedron, 26, 4542-4550 (2007). 